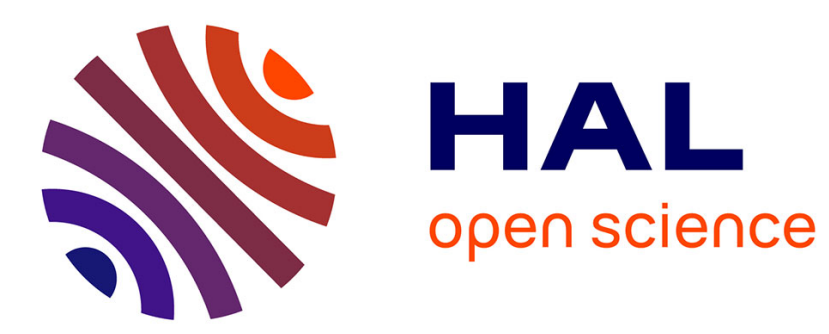

\title{
La mortalité maternelle en milieu rural sénégalais : l'expérience du nouvel hôpital de Ninéfescha
}

\author{
Almamy-Malick Kante, Gilles Pison
}

\section{To cite this version:}

Almamy-Malick Kante, Gilles Pison. La mortalité maternelle en milieu rural sénégalais : l'expérience du nouvel hôpital de Ninéfescha. Population (édition française), 2010, 65 (4), pp.753-780. 10.3917/popu.1004.0753 . hal-02081787

\section{HAL Id: hal-02081787 https://hal.science/hal-02081787}

Submitted on 27 Mar 2019

HAL is a multi-disciplinary open access archive for the deposit and dissemination of scientific research documents, whether they are published or not. The documents may come from teaching and research institutions in France or abroad, or from public or private research centers.
L'archive ouverte pluridisciplinaire $\mathbf{H A L}$, est destinée au dépôt et à la diffusion de documents scientifiques de niveau recherche, publiés ou non, émanant des établissements d'enseignement et de recherche français ou étrangers, des laboratoires publics ou privés. 


\title{
Almamy Malick KANTÉ* et Gilles PISON*
}

\section{La mortalité maternelle en milieu rural sénégalais. L'expérience du nouvel hôpital de Ninéfescha}

\begin{abstract}
On attribue souvent la baisse de la mortalité à l'amélioration de la quantité et de la qualité de l'offre de soins, une offre dans I'ensemble encore très insuffisante en Afrique subsaharienne où I'on enregistre toujours les niveaux de mortalité les plus élevés du monde. Augmenter l'offre de santé dans cette région suffirait-il à assurer un meilleur accès aux soins et à accélérer le déclin de la mortalité ? C'est la question qu'abordent Almamy Malick KANTÉ et Gilles PIsON en étudiant l'impact de l'installation d'un hôpital moderne sur les comportements de la population dans une zone rurale et isolée du Sénégal. Il s'avère que quatre ans après son ouverture, les comportements de la population n'ont guère évolué : le recours à l'hôpital pour les visites prénatales et les accouchements demeure faible, la mortalité maternelle toujours très élevée. Un exemple, selon les auteurs, de l'inadéquation entre l'offre de soins de cette structure hospitalière et les besoins de la population.
\end{abstract}

En Afrique, plus qu'ailleurs, l'offre de soins reste insuffisante. La construction de nouvelles infrastructures sanitaires est une priorité, mais suffit-elle à améliorer la santé de la population ? La question se pose lorsque de nouvelles installations sanitaires sont construites sans que les populations auxquelles elles sont destinées les utilisent pleinement, et sans que cela soit immédiatement suivi d'une amélioration des indicateurs de santé. Comment alors expliquer la lenteur des changements? Provient-elle de l'inadéquation entre l'offre et les besoins? de «freins culturels » empêchant la diffusion de méthodes modernes? Nous examinons ici les facteurs en cause dans le cas de l'implantation d'un nouvel hôpital au cœur d'une région rurale du Sénégal mal équipée jusqu'alors, la zone de Bandafassi.

\footnotetext{
*Institut national d'études démographiques, Paris.

Correspondance : Gilles Pison, Institut national d'études démographiques, 133 bd Davout, 75980 Paris Cedex 20, tél : 01560621 26, courriel : gilles.pison@ined.fr
} 
Ce nouvel équipement avait pour premier objectif de faire reculer la mortalité maternelle dans la région en offrant des soins de haut niveau, notamment en obstétrique. La population de la région ayant fait l'objet d'une surveillance démographique depuis le début des années 1970, nous avons pu retracer de façon précise l'évolution de la mortalité dans la population depuis cette période. Nous avons pu vérifier notamment si l'implantation de cet hôpital avait ou non fait reculer la mortalité, notamment maternelle. Pour mieux comprendre les raisons de ces succès ou ces échecs, nous avons mené plusieurs enquêtes, qualitatives et quantitatives, sur la perception du nouvel hôpital par la population de cette région, leurs comportements de recours aux soins pendant la grossesse et lors de l'accouchement.

\section{Que sait-on de l'accès aux soins et de la santé des femmes en Afrique subsaharienne?}

L'Afrique subsaharienne est la région du monde où la santé est la plus précaire, quel que soit l'indicateur retenu pour la mesurer. Malgré une baisse substantielle au cours du dernier demi-siècle, la mortalité des enfants y atteint l'un des niveaux les plus élevés au monde (Hill, 1989 ; Barbieri et Vallin, 1996 ; Tabutin et Schoumaker, 2004). D’après les Nations unies, 148 nouveau-nés sur 1000 y sont morts avant d'atteindre l'âge de 5 ans au cours de la période 20052009 (Nations unies, 2009). Le taux de mortalité maternelle y serait de 820 décès maternels pour 100000 naissances vivantes en 2005, et il n'aurait pas baissé sensiblement au cours des deux dernières décennies - il était estimé à 830 pour 100000 en 1990 (OMS, 2007). L'offre de soins y est limitée : bien que représentant un huitième de la population mondiale en 2005 (12\%), l'Afrique subsaharienne ne dispose que de $3 \%$ du personnel sanitaire et de moins de $1 \%$ des ressources financières mondiales (OMS, 2006).

Augmenter l'offre de soins en Afrique est certes nécessaire, mais est-ce suffisant pour assurer l'accès universel à la santé ? Pour que les services de soins soient utilisés, il faut en assurer l'accessibilité économique (Diallo, 2003 ; Commeyras et Ndo, 2003) et géographique (Vigneron, 2001), mais aussi l'accompagnement socioculturel (Caldwell, 1979 ; Jaffré et Olivier de Sardan, 2003 ; Gastineau, 2003). Différents facteurs, d'ordre individuel ou collectif, liés au ménage ou à l'environnement social et économique, influent sur les comportements en matière de soins (Caldwell, 1979 ; Mosley et Chen, 1984 ; Palloni, 1985). Même lorsque des services de santé existent, certaines franges de la population ne les utilisent pas forcément, ou uniquement en cas d'extrême urgence.

Une des mesures préconisées pour réduire la mortalité maternelle est d'inciter les femmes à accoucher en maternité. La tradition est souvent d'accoucher à domicile en Afrique subsaharienne (Cantrelle et Locoh, 1990). Mais les pratiques varient d'une région, d'une ethnie à l'autre. Chez les Peul de la région du Fouta Djallon, en Guinée, les accouchements ont lieu à domicile, sans aucune assistance, « la coutume étant la femme seule avec Dieu » (Cantrelle 
et al., 1992). Chez les Peul de la région de Salémata, au Sénégal, l'accouchement a lieu à l'extérieur de la concession ${ }^{(1)}$, près de la véranda. La femme qui accouche est aidée par les autres femmes de la concession familiale (Lestrange et PassotGuevara, 1981). Chez les Diola de la région de Mlomp (Sénégal), les femmes au contraire n'accouchent pas chez elles : avant la construction en 1968 d'une maternité villageoise moderne dans la région, les femmes accouchaient dans une kalamba, bâtiment construit à l'écart des habitations, strictement interdit aux hommes, et servant uniquement aux accouchements, une sorte de « maternité traditionnelle » (Énel et al., 1993). Chez les Sereer du Sénégal, « l'accouchement est une étape initiatique de la vie de la femme qui se doit d'enfanter seule et en silence. Ce stoïcisme étant la condition pour passer honorablement du statut d'épouse à celui de mère » (Kalis, 1984, cité par Leroy et Garenne, 1989).

\section{La population de Bandafassi : méthodes d'étude}

La zone choisie pour l'étude est celle de Bandafassi au sud-est du Sénégal, à $750 \mathrm{~km}$ de Dakar (figure 1). Elle comprend 42 villages répartis sur un territoire d'environ $600 \mathrm{~km}^{2}$. La population, de 12079 habitants en 2007, est divisée en trois groupes ethniques résidant dans des villages séparés : les Bedik (26\% des habitants), les Malinké (16\%) et les Peul (58\%). Les villages malinké sont situés dans la partie Nord de la zone d'étude, les villages peul dans la partie Sud, les villages bedik se répartissent dans l'ensemble de la zone (figure 1).

\section{L'offre sanitaire à Bandafassi}

L'offre sanitaire a sensiblement changé dans la région de Bandafassi suite à l'ouverture de l'hôpital de Ninéfescha en 2003. Nous allons donc analyser séparément ces deux périodes.

\section{La situation avant 2003}

La population étudiée bénéficiait jusqu'en 2003 de deux types d'offre sanitaire, l'une publique et l'autre privée, qui ont continué de coexister après 2003 :

- Loffre sanitaire publique consiste en deux dispensaires tenus chacun par un infirmier, l'un situé dans le village de Bandafassi, à l'intérieur de la zone d'étude, et l'autre, dans celui de Tomborokoto, en dehors de la zone. La majorité des villages de la zone d'étude dépendent du dispensaire de Bandafassi. L'hôpital le plus proche est celui de la ville de Kedougou (près de 19000 habitants en 2007), située à une distance de 12 km à $40 \mathrm{~km}$ selon les villages.

- Loffre sanitaire privée est assurée par la mission catholique de Kedougou qui prodigue des soins de santé dans les villages malinké et bedik situés dans le Nord de la zone d'étude via une équipe mobile visitant périodiquement ces villages (figure 1).

(1) Espace correspondant à une unité d'habitation occupée par un ménage ou une famille élargie. 
Figure 1. Carte de la zone d'étude de Bandafassi, situation en 2007
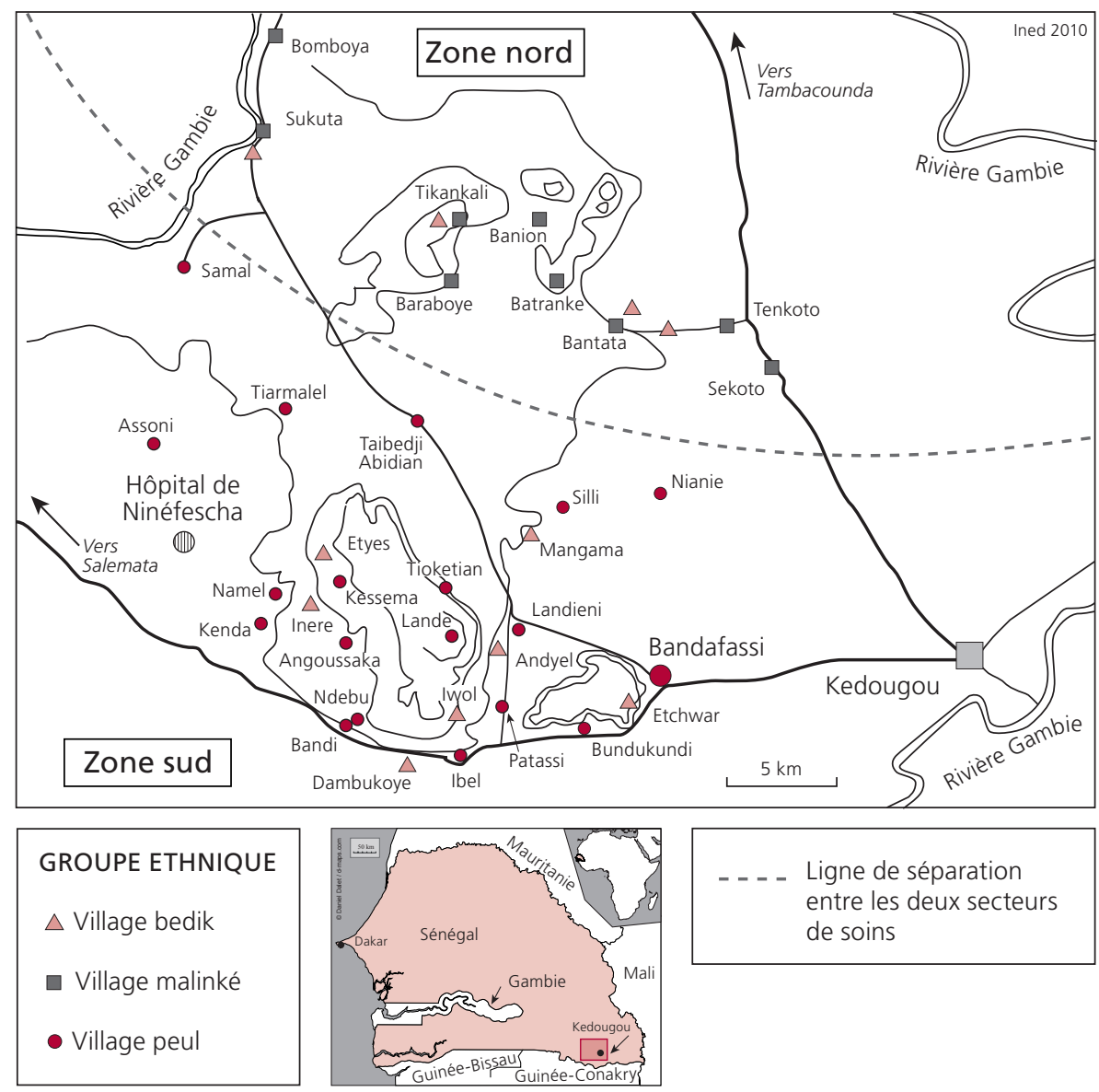

Ligne de séparation entre les deux secteurs de soins

Des cases de santé ont par ailleurs été installées progressivement dans plusieurs villages à partir des années 1980, certaines existant avant même la promotion de ce type d'équipement à l'échelle mondiale (notamment suite à la conférence d'Alma-Ata). Elles fonctionnent sous la responsabilité d'agents de santé communautaires ${ }^{(2)}$. Les habitants consultent également des praticiens traditionnels, guérisseurs souvent spécialisés dans le traitement de symptômes particuliers et proposant des remèdes traditionnels (Kanté, 2009).

Les accouchements ont lieu pour la plupart à domicile, de la façon décrite depuis plusieurs décennies déjà. Chez les Bedik par exemple, la femme accouche

(2) Ils sont aussi appelés « hygiénistes » ou " auxiliaires sanitaires ». Habitants des villages, ils ont reçu une formation de base leur permettant d'assurer les soins primaires (traitement des petites blessures, distribution de médicaments utilisables en automédication, etc.). Les cases de santé ne sont pas équipées pour des consultations prénatales. 
dans sa case et le mari attend à l'extérieur, «C'est le seul homme admis à rester dans le carré... La femme ne criera jamais même si ça fait très mal... Toutes les femmes expérimentées du village - et non seulement celles du carré - peuvent aider à l'accouchement » (Gomila et Clarkson, 1983).

Jusqu'en 2003, lorsqu'une femme rencontrait des difficultés pour accoucher et avait besoin d'être évacuée d'urgence à l'hôpital pour être opérée d'une césarienne, l'hôpital le plus proche pour ce type d'intervention était situé dans la capitale régionale, Tambacounda, à $250 \mathrm{~km}$. Certaines femmes étaient évacuées directement à Dakar, à $750 \mathrm{~km}$. Le mauvais état des routes, souvent impraticables pendant la saison des pluies (de juin à octobre), ajoutait aux difficultés liées à la distance et rendait problématique les évacuations d'urgence (Pison et al., 2000). L'hôpital de Kedougou a bénéficié depuis le milieu des années 1990 d'équipements et de personnel pour pouvoir effectuer des césariennes, grâce notamment à un programme de formation à ce type d'intervention pour les médecins généralistes et anesthésistes. Ce plateau opératoire n'a cependant fonctionné que de façon épisodique depuis son ouverture, les femmes ayant besoin d'être opérées continuent pour la plupart à être évacuées à Tambacounda ou Dakar.

\section{La situation après l'ouverture de l'hôpital de Ninéfescha en 2003}

En 2003, l'offre sanitaire dans la région a été enrichie par l'ouverture d'un nouvel hôpital moderne. Cet hôpital offre des services de médecine générale, de gynécologie obstétrique et de chirurgie comme on n'en trouve que dans les hôpitaux les plus importants des grandes villes ${ }^{(3)}$. Il peut satisfaire notamment toutes sortes d'urgences sanitaires. Sa construction avait pour premier objectif de réduire la mortalité maternelle dans la région (Kanté, 2007).

L'hôpital a été construit en pleine campagne, au milieu des collines, à $35 \mathrm{~km}$ de Kedougou, à côté du hameau bedik de Ninéfescha (figure 1). Le site a été choisi en suivant les recommandations d'un missionnaire ${ }^{(4)}$ ayant longtemps travaillé auprès des villages bedik de la région. Son souci était d'abord d'améliorer les conditions sanitaires du sous-groupe bedik, jugées moins bonnes que celles des autres groupes ethniques.

Il faut noter que l'installation de l'hôpital de Ninéfescha n'a pas été accompagnée d'une réorganisation de l'offre de soins des infrastructures préexistantes dans la région ; les infirmiers des dispensaires de Bandafassi et de la mission catholique de Kedougou sont toujours restés en charge de leur secteur de soins.

Pour étudier l'impact du nouvel hôpital sur la santé de la population, en particulier la mortalité maternelle, et examiner quel usage les habitants de la

(3) Cet hôpital a été construit à l'initiative de la « Fondation Éducation-Santé » de Viviane Wade, épouse du Président de la république du Sénégal ; sa construction a été financée par le Conseil général des Hauts-de-Seine en France.

(4) Xavier Gobaille, missionnaire de la congrégation des Pères du Saint-Esprit. Il a vécu 17 ans dans la région (de 1983 à 2000), et s'est principalement occupé des villages bedik en organisant notamment pour eux la construction de puits et l'établissement de cases de santé. 
région ont fait de cette nouvelle infrastructure, nous avons analysé les données de suivi démographique et organisé plusieurs enquêtes sur les conditions de grossesse et d'accouchement, ainsi que les comportements de recours aux soins.

\section{La collecte de données}

\section{L'observation démographique suivie}

La population de la zone d'étude de Bandafassi fait l'objet d'une observation démographique suivie par enquête à passages répétés depuis 1970 (Pison, 1982, 2006 ; Pison et al., 2002). Après un premier recensement, les villages ont été visités chaque année. À l'occasion de chaque visite, les ménages sont passés en revue, la liste des personnes présentes dans chaque ménage lors de la visite précédente est vérifiée et des informations sur les naissances, mariages, migrations et décès (y compris leur cause) survenus depuis sont recueillies. L'observation n'a pas commencé au même moment dans les différents villages. À son début, en 1970, le suivi portait uniquement sur la sous-population des villages malinké. Il a été étendu aux autres villages en deux étapes : en 1975, les villages peul ont été ajoutés, puis en 1980, les villages bedik.

Comme dans beaucoup de régions rurales d'Afrique, la majorité des décès ont lieu sans qu'un médecin ait vu le malade avant sa mort ou qu'une autopsie ait pu être effectuée après. Pour déterminer les causes de décès, des informations sont recueillies en interrogeant les proches de la personne décédée sur les circonstances du décès et les symptômes de la maladie l'ayant précédée. Jusqu'en 1984, l'entretien se faisait de façon libre. Depuis, il utilise un questionnaire d'autopsie verbale $^{(5)}$ (Garenne et Fontaine, 1988 ; Desgrées du Loû et al., 1996).

Les informations recueillies directement auprès des familles sont complétées par celles figurant éventuellement dans les registres des dispensaires ou des hôpitaux de la région, lorsque la personne y est décédée ou y a séjourné avant son décès. L'ensemble des informations ainsi rassemblées sont soumises indépendamment à un ou plusieurs médecins qui portent un diagnostic sur la cause du décès ${ }^{(6)}$. Les décès maternels sont repérés par référence à la définition de la mort maternelle des $9^{\mathrm{e}}$ et $10^{\mathrm{e}}$ révisions de la Classification internationale des maladies (CIM) : « La mort maternelle se définit comme le décès d'une femme survenu au cours de la grossesse ou dans un délai de 42 jours après sa terminaison, quelle qu'en soit la durée ou la localisation, pour une cause quelconque déterminée ou aggravée par la grossesse ou les soins qu'elle a motivés, mais ni accidentelle, ni fortuite » (OMS, 1977 ; OMS, 1993).

(5) Les biais éventuels sont nombreux (biais dans l'enregistrement des décès, biais dans la détermination des causes de décès, etc.). Cela dit, le contexte est favorable ici en raison des méthodes d'observation utilisées. Les biais sont au total d'importance moindre que dans beaucoup d'études et de statistiques.

(6) La recommandation est en général d'effectuer deux lectures indépendantes et une troisième si les deux premières ne sont pas concordantes. 
Les données de surveillance démographique ont permis d'estimer l'espérance de vie à la naissance à 53 ans, sexes confondus, au cours de la période 2000-2006, et le risque pour un nouveau-né de mourir avant 5 ans à $189 \%$ (tableau annexe 1). La fécondité est de 7,0 naissances vivantes en moyenne par femme au cours de cette même période, avec peu de différences d'une ethnie à l'autre (tableau annexe 1 ; Guyavarch, 2003).

\section{Les enquêtes sur le recours aux soins}

L'enquête pilote sur la perception et l'utilisation de l'hôpital de Ninéfescha (2005)

À l'occasion du passage démographique annuel de 2005, 50 familles dont l'un des membres était décédé au cours des 12 derniers mois ont été interrogées. Ces familles devaient de toute façon faire l'objet d'une enquête d'autopsie verbale pour déterminer la cause du décès ; elles ont aussi été questionnées de façon détaillée sur le recours à l'hôpital de Ninéfescha lors de la maladie ayant précédé le décès, ainsi que sur leur perception de cet hôpital. Ces interviews ont été menées en utilisant un questionnaire semi-directif. La même année, dix entretiens également semi-directifs ont été organisés avec des agents de santé et des responsables villageois sur la perception et l'utilisation de l'hôpital de Ninéfescha, ainsi que les problèmes, besoins et attentes des habitants face à la nouvelle infrastructure sanitaire.

L'enquête sur le recours aux soins à Bandafassi (2007)

L'enquête pilote de 2005 était le préambule à une enquête plus large, en population générale, menée en mars 2007 (Kanté et al., 2008). L'enquête visait à décrire les comportements en matière de santé, mesurer la place de l'hôpital de Ninéfescha dans les itinéraires thérapeutiques et analyser les variations socioéconomiques, démographiques et géographiques des recours aux soins (Kanté, 2009). Elle comportait un volet sur la grossesse et l'accouchement avec l'interview d'un échantillon aléatoire d'environ la moitié des femmes ayant accouché dans les 12 derniers mois. Les femmes de l'échantillon ont été sélectionnées en choisissant dans le cahier d'enquête de chaque village la première naissance enregistrée, puis la troisième, la sixième, etc. Cela a abouti à une sélection de 253 femmes sur les 579 ayant accouché entre les passages des années 2006 et 2007. Lorsqu'une femme sélectionnée était absente du village le jour de l'enquête, elle était remplacée dans l'échantillon par une autre femme de la même concession ou d'une concession voisine ayant également accouché. Quatorze femmes absentes n'ayant pu être remplacées (5,5\%), l'étude porte finalement sur 239 femmes.

\section{La méthode d'analyse}

Les différences économiques sont peu marquées au sein de la population étudiée $^{(7)}$. Toutefois, les comportements sanitaires peuvent varier d'une ethnie

(7) À l'exception de la maison de l'infirmier et des fonctionnaires de la sous-préfecture de Bandafassi, les habitants vivent dans des cases avec des murs en terre (bauge, adobe, torchis) et des toits généralement en paille. 
à l'autre et dépendre de la proximité du village par rapport au centre de soins, de sa plus ou moins grande facilité d'accès en fonction notamment de sa localisation en plaine ou en altitude ${ }^{(8)}$.

Elle est mesurée par deux variables. La première indique la situation géographique du village de résidence par rapport au dispensaire de Bandafassi, en distinguant trois situations selon que le village relève : 1) du système de santé public et est situé en plaine à moins de $15 \mathrm{~km}$ du dispensaire de Bandafassi ; 2) du système de santé public et situé en altitude, ou en plaine mais à plus de $15 \mathrm{~km}$ du dispensaire de Bandafassi ; 3) du système de santé privé. La seconde variable indique l'éloignement de l'hôpital de Ninéfescha, en distinguant les villages situés à moins de $15 \mathrm{~km}$ de l'hôpital des autres villages, situés à $15 \mathrm{~km}$ ou plus.

D'autres facteurs tels que le groupe d'âges, le rang de la grossesse, le fait d'avoir été scolarisé ou de disposer de moyens de transport, celui d'écouter les médias ${ }^{(9)}$ dans le ménage pourraient influer sur les recours aux centres de santé.

Nous procédons d'abord à une analyse des données de surveillance démographique, afin de retracer l'évolution de la mortalité maternelle dans la région en lien avec l'ouverture de l'hôpital de Ninéfescha.

Utilisant les données de l'enquête sur le recours aux soins, nous étudions ensuite les déterminants de la consultation prénatale ou de l'accouchement en maternité dans la région. Nous classons ici les femmes en deux catégories: celles qui n'ont pas consulté de personnel de santé et celles qui l'ont consulté au moins une fois, lors de leur dernière grossesse pour une visite prénatale et/ou pour un accouchement en maternité. Dans un premier temps, nous examinons de façon séparée les relations entre chaque caractéristique des femmes, des ménages, des villages, et la propension à consulter (analyse univariée). Puis nous analysons l'effet conjoint des différents facteurs grâce à des régressions logistiques multivariées. Pour cela, nous introduisons dans le modèle uniquement les variables significatives au seuil de $10 \%$ dans l'analyse univariée, en éliminant certaines d'entre elles pour des raisons de colinéarité (voir plus loin). Notre variable d'intérêt est ici la proximité des villages par rapport à l'hôpital de Ninéfescha, elle est introduite dans le modèle quel que soit son degré de significativité dans l'analyse univariée. Enfin, nous effectuons une analyse du contenu des entretiens afin de mieux saisir les comportements de recours aux soins des femmes enceintes de la région.

(8) La région de Bandafassi comporte des reliefs avec des collines et des escarpements en haut desquels sont localisés la plupart des villages bedik et quelques villages peul et malinké. Les points GPS pris en 2005 permettent de classer les villages de la zone en deux groupes : les villages de plaine et ceux d'altitude (Kanté, 2009).

(9) L'écoute des émissions sur la santé diffusées par la radio communautaire pourrait entraîner un recours plus fréquent au centre de santé par les femmes. 


\section{Résultats}

\section{Niveaux et évolution de la mortalité maternelle}

Le taux de mortalité maternelle est de 911 décès pour 100000 naissances vivantes en moyenne au cours de la période 1976-2006 (tableau 1). Il ne montre pas de signe de baisse à partir de 2003, ou s'il a diminué, c'est de façon insuffisante pour que nous puissions le déceler ${ }^{(10)}$, le taux passant de 1010 en 1999-2002 à 915 en 2003-2006. Ces taux sont comparables à ceux de précédentes études menées dans plusieurs régions rurales du Sénégal (Pison et al., 2000) et de l'Afrique de l'Ouest (Ronsmans et al., 2003). À noter qu'avec ces chiffres, Bandafassi a une mortalité maternelle deux fois plus élevée que la moyenne nationale en zone rurale telle qu'estimée par la dernière enquête démographique et de santé au Sénégal (472 pour 100000 pour la période 19982005), la moyenne nationale générale, campagnes et villes confondues, étant de 401 pour 100000 (Ndiaye et al., 2006).

Tableau 1. Caractéristiques de la mortalité maternelle à Bandafassi, 1976-2006

\begin{tabular}{|c|c|c|c|c|}
\hline \multirow[b]{2}{*}{ Période d'étude } & \multicolumn{2}{|c|}{ Effectifs } & \multirow{2}{*}{$\begin{array}{l}\text { Taux de } \\
\text { mortalité } \\
\text { maternelle }\end{array}$} & \multirow{2}{*}{$\begin{array}{l}\text { Intervalle de } \\
\text { confiance } \\
\text { (à } 95 \%)\end{array}$} \\
\hline & Décès maternels & $\begin{array}{c}\text { Naissances } \\
\text { vivantes }\end{array}$ & & \\
\hline 1976-2002 dont & 91 & 9999 & 910 & [723 - 1 097] \\
\hline 1976-1998 & 71 & 8019 & 885 & [679- 1 091] \\
\hline $1999-2002^{(b)}$ & 20 & 1980 & 1010 & {$[567-1453]$} \\
\hline $2003-2006^{(c)}$ & 19 & 2076 & 915 & {$[504-1$ 327] } \\
\hline $1976-2006$ & 110 & 12075 & 911 & {$[740-1081]$} \\
\hline \multicolumn{5}{|c|}{$\begin{array}{l}\text { (a) Nombre de décès maternels pour } 100000 \text { naissances vivantes. } \\
\text { (b) Période des } 4 \text { années précédant l'ouverture de l'hôpital de Ninéfescha. } \\
\text { (c) Période des } 4 \text { années suivant l'ouverture de l'hôpital de Ninéfescha. } \\
\text { Source : Base de données de Bandafassi (Pison et al., } 2002 \text {; Kanté, 2009). }\end{array}$} \\
\hline
\end{tabular}

Pour comprendre les raisons du faible impact de l'hôpital de Ninéfescha sur la mortalité maternelle, nous analysons les informations recueillies par les enquêtes sur le recours aux soins de 2005 et 2007.

\section{Les recours aux soins chez les femmes enceintes}

\section{Caractéristiques des femmes de l'enquête de 2007}

Les 239 femmes interrogées en 2007, qui avaient accouché dans les 12 derniers mois, comptent $58 \%$ de Peul, $24 \%$ de Bedik et $17 \%$ de Malinké, soit une répartition correspondant à celle de la population générale, la fécondité étant

(10) Un calcul permet d'estimer de combien la baisse aurait dû être pour être significative, compte tenu des effectifs concernés. Par exemple, en estimant la mortalité maternelle avant 2003 à $910 / 100000$ (=0,009), avec 2076 naissances vivantes au cours de la période 2003-2006, on a un intervalle de confiance autour de la valeur 0,009 de l'ordre de $[0,005 ; 0,013]$. Pour être significative, la baisse du taux de mortalité maternelle aurait donc dû être de presque la moitié. 
similaire dans les trois groupes ethniques. Au moment de l'enquête, $53 \%$ des femmes sont en union monogame, $43 \%$ en union polygame et $4 \%$ ne sont pas mariées (célibataires, veuves ou divorcées). Pour $10 \%$ des femmes, il s'agit de leur première grossesse. Le quart des femmes (25\%) est allé à l'école française au moins une année et $40 \%$ déclarent savoir lire dans une langue quelconque (français ou langue locale : pulaar, bedik ou mandinka). Près de $85 \%$ des femmes écoutent la radio au moins une fois par semaine. Un tiers des femmes vivent dans un ménage peu équipé, ne disposant ni de vélo, ni de radio, ni de moustiquaire. La moitié réside dans un village disposant d'un agent de santé, près de $35 \%$ habitent à moins de $15 \mathrm{~km}$ du dispensaire de Bandafassi, 20 \% à moins de $15 \mathrm{~km}$ de l'hôpital de Ninéfescha ${ }^{(11)}$ et 40 \% résident dans les villages localisés en altitude et difficiles d'accès (tableau annexe 2).

\section{Déroulement de la grossesse}

Les soins pendant la grossesse

Durant leur grossesse, $17 \%$ des femmes n'ont consulté aucun personnel de santé et 83 \% l'ont consulté au moins une fois (tableau annexe 3). Pour $44 \%$ de ces dernières, la consultation a eu lieu dans leur village même, lors de visites mensuelles de l'infirmier, 38 \% se sont déplacées au dispensaire de Bandafassi, 9 \% à l'hôpital de Ninéfescha, et les $9 \%$ restants dans les autres centres de santé ${ }^{(12)}$ de la région. À noter que près de $10 \%$ des femmes ayant consulté un personnel de santé ont effectué en parallèle une consultation chez le guérisseur.

Les facteurs influant sur la probabilité d'avoir une consultation prénatale

Les caractéristiques telles que l'ethnie, la religion, le groupe d'âges, le niveau d'instruction, l'écoute de la radio, le type de ménage et la situation du village par rapport au dispensaire de Bandafassi sont associées à des variations de la probabilité qu'une femme effectue une consultation lors de sa grossesse (tableau annexe 4). Toutefois, certains de ces facteurs sont colinéaires deux à deux et ils ne sont pas introduits conjointement dans le modèle. Il s'agit notamment de l'ethnie et de la religion; de la pratique de la lecture et de la fréquentation de l'école publique, de l'école coranique et des cours d'alphabétisation; de l'écoute de la radio et de l'utilisation du téléphone (annexe 5). Notre choix porte par exemple sur la fréquence de l'écoute de la radio plutôt que celle de l'utilisation du téléphone portable, dans la mesure où la radio reste un des biens les plus répandus dans la zone (près de $75 \%$ des ménages enquêtés possèdent au moins une radio).

Finalement, les facteurs tels que l'ethnie, le niveau d'instruction et le village de résidence, sont associés à des variations de la probabilité de consulter pendant la grossesse (tableau 2). Les femmes malinké et peul se rendent davantage à la consultation prénatale que les femmes bedik. De même, les femmes ayant été scolarisées consultent plus fréquemment pendant la grossesse. Enfin, les

(11) Cette répartition est voisine de celle de l'ensemble de la population de la zone d'étude de Bandafassi.

(12) Au dispensaire de Tomborokoto, à l'hôpital de Kedougou ou à l'hôpital de Tambacounda. 


\section{Conditions de l'accouchement}

Le lieu d'accouchement

Parmi les 239 femmes interrogées en 2007, 86 \% ont accouché à domicile (dans la concession, les champs, etc.) contre 14 \% en maternité (6 \% à l'hôpital de Ninéfescha, $4 \%$ au dispensaire de Bandafassi et $4 \%$ dans un autre centre de santé de la région).

Les facteurs influant sur la probabilité d'accoucher en maternité

Les femmes résidant dans les villages proches du dispensaire de Bandafassi accouchent plus fréquemment en maternité que les autres quelle que soit la maternité ; il en est de même pour les femmes scolarisées et les primipares. Enfin, les femmes dont l'accouchement a présenté des difficultés ont plus de chances d'avoir été transférées dans un centre de santé (tableau 3). En revanche, celles qui habitent à proximité de l'hôpital de Ninéfescha n'y accouchent pas beaucoup plus que celles qui en sont éloignées.

Tableau 3. Facteurs influençant l'accouchement en maternité à Bandafassi, analyse par régression multivariée

\begin{tabular}{|c|c|c|c|c|c|}
\hline Variable & Modalité & $\begin{array}{c}\text { OR } \\
\text { [IC à } 95 \%]\end{array}$ & $\begin{array}{c}p \\
\text { bilatéral }\end{array}$ & $\begin{array}{c}\text { OR } \\
\text { [IC à } 90 \%]\end{array}$ & $\begin{array}{c}p \\
\text { unilatéral }\end{array}$ \\
\hline Rang de la grossesse & Primipare / Autre & $3,8[1,2-12,0]$ & $* *$ & $3,8[1,5-10,0]$ & ** \\
\hline $\begin{array}{l}\text { Fréquentation de } \\
\text { l'école publique }\end{array}$ & Oui / Non & $3,2[1,3-8,0]$ & ** & $3,2[1,5-6,9]$ & $* * *$ \\
\hline Écoute de la radio & Oui / Non & $1,3[0,5-3,6]$ & ns & $1,3[0,6-3,1]$ & ns \\
\hline $\begin{array}{l}\text { Complication à } \\
\text { l'accouchement }\end{array}$ & Oui / Non & $4,9[1,8-13,0]$ & $* * *$ & $4,9[2,2-11,1]$ & $* * *$ \\
\hline $\begin{array}{l}\text { Lien avec le chef de } \\
\text { concession }\end{array}$ & Femme / Autre & $2,5[1,0-6,3]$ & * & $2,5[1,2-5,4]$ & ** \\
\hline $\begin{array}{l}\text { Système de santé du } \\
\text { village par rapport au }\end{array}$ & $\begin{array}{l}\text { Public en plaine }{ }^{(b)} / \\
\text { Public en altitude }\end{array}$ & $6,9[2,4-20,3]$ & $\star * *$ & $6,9[2,8-17,1]$ & $* * *$ \\
\hline $\begin{array}{l}\text { dispensaire de } \\
\text { Bandafassi }^{(a)}\end{array}$ & $\begin{array}{l}\text { Privé / Public en } \\
\text { altitude }^{(c)}\end{array}$ & $1,0[0,3-3,9]$ & ns & $1,0[0,3-3,1]$ & ns \\
\hline $\begin{array}{l}\text { Situation du village par } \\
\text { rapport à l'hôpital de } \\
\text { Ninéfescha }{ }^{(a)}\end{array}$ & $\begin{array}{l}\text { Moins de } 15 \mathrm{~km} / \\
\text { plus de } 15 \mathrm{~km}^{(\mathrm{d})}\end{array}$ & $0,6[0,2-1,8]$ & ns & $0,6[0,2-1,4]$ & ns \\
\hline \multicolumn{6}{|c|}{$\begin{array}{l}\text { Nombre d'observations }=239 . \\
\text { Significativité statistique : }{ }^{* *} p<0,01 ;{ }^{* *} p<0,05 ;{ }^{*} p<0,10 ; n s=\text { non significatif. } \\
\text { (a) Ces variables sont proches. Elles ont été introduites pour évaluer l'effet de la distance sur la probabilité de } \\
\text { l'accouchement en maternité. } \\
\text { (b) Public en plaine et à moins de } 15 \mathrm{~km} \text { du dispensaire de Bandafassi. } \\
\text { (c) Public en altitude ou à plus de } 15 \mathrm{~km} \text { du dispensaire de Bandafassi. } \\
\text { (d) Plus de } 15 \mathrm{~km} \text { de l'hôpital de Ninéfescha ou en altitude. } \\
\text { Source : Enquête sur le recours aux soins à Bandafassi, } 2007\end{array}$} \\
\hline
\end{tabular}

\section{Les freins à la fréquentation de l'hôpital de Ninéfescha}

Les entretiens menés en 2005, soit deux ans après l'ouverture de l'hôpital de Ninéfescha, révèlent qu'à l'époque la grande majorité des personnes interrogées déclaraient vouloir se faire soigner dans cet hôpital, même si elles allaient 
pourtant consulter ailleurs, généralement au dispensaire de Bandafassi ou à l'hôpital de Kedougou. Les obstacles qu'elles mentionnaient comme les empêchant d'aller à l'hôpital de Ninéfescha, étaient en premier lieu les problèmes de nourriture sur place (32\% des personnes interrogées), et de logement pour les personnes accompagnant le malade (28\%). Dans ces régions, lorsqu'une famille se rend en ville pour accompagner un malade à l'hôpital, elle trouve en général un hébergement chez des parents ou des connaissances. Ce type d'entraide n'était pas possible à l'époque dans le cas de l'hôpital de Ninéfescha, ce dernier ayant été construit en pleine campagne avec seulement un petit hameau bedik à côté qui ne comportait que quelques cases. Malgré les traditions d'hospitalité toujours en vigueur, leurs habitants n'avaient ni les capacités ni la vocation à accueillir un grand nombre de familles qui leur étaient, par ailleurs, pour la plupart étrangères. Parmi les autres difficultés sont mentionnés, en 2005, le coût élevé des médicaments (cité par 25 \% des personnes interrogées) et le manque d'argent (13\%). À l'époque, le tarif d'admission à l'hôpital et les coûts des soins étaient calqués sur ceux pratiqués par les grands hôpitaux des villes; ils étaient nettement supérieurs à ceux pratiqués par les dispensaires de la région sachant qu'un accouchement était normalement " gratuit » partout au Sénégal depuis 2004 (Witter et al., 2008). Autre obstacle à la fréquentation de l'hôpital dans les premières années de son ouverture, l'idée fausse selon laquelle l'hôpital avait été construit pour les seuls Bedik (citée par 3 \% des personnes interrogées) (Kanté, 2007).

Pour remédier aux obstacles que notre enquête pilote de 2005 avait permis d'identifier, les responsables de l'hôpital ont organisé des campagnes d'information dans les villages de la région afin de démentir les idées fausses et inciter les habitants à venir consulter. Ils ont également revu à la baisse les tarifs d'admission et de soins pour les ramener aux niveaux pratiqués dans les dispensaires de la région ou à des niveaux proches. En 2007, la consultation était de 200 FCFA dans les dispensaires et de 300 FCFA à l'hôpital de Ninéfescha ${ }^{(15)}$. Enfin, un hébergement et des possibilités de nourriture sur place ont été organisés pour les familles de malades.

D'autres freins à la fréquentation de l'hôpital de Ninéfescha sont apparus lors des entretiens menés en 2005 et de l'enquête de 2007 auprès de l'échantillon de femmes ayant accouché dans les 12 derniers mois. Une femme du village même de Ninéfescha témoigne ainsi : « je n'ai pas accouché à l'hôpital de Ninéfescha car là-bas le médecin t'ouvre pour rien... ». La moitié des femmes de la population qui ont accouché à l'hôpital de Ninéfescha en 2006 ont de fait subi une césarienne (Kanté, 2009). L'accès difficile à l'hôpital semble jouer lui aussi. En discutant avec un groupe de femmes du village de Samal, situé en plaine et à moins de $15 \mathrm{~km}$ de Ninéfescha, ces dernières déclaraient : « ...aller jusqu'à l'hôpital de Ninéfescha pour les accouchements [rires] mais il n'y a pas

(15) 100 FCFA équivalaient à 0,15 euro en 2007 ; 300 FCFA correspond au prix du plat de base dans les restaurants de la région (riz au poisson ou à la viande) en 2007. 
de bonne route pour ça...». D’autres facteurs peuvent avoir également joué, comme en témoigne une femme du village de Ndébu, situé en plaine à moins de $10 \mathrm{~km}$ : « ...les habitants disent que lorsqu'un nouveau hôpital est ouvert dans une zone il y a toujours des sacrifices humains et ça touche les premiers consultants, c'est pour ça on n'y va pas ».

Si les habitants des villages situés aux alentours de l'hôpital de Ninéfescha le fréquentent peu, ce dernier est pourtant actif, ayant trouvé une clientèle chez les habitants de la ville voisine de Kedougou. Ceux-ci n'hésitent pas à se déplacer pour venir profiter des compétences spécialisées offertes par l'hôpital de Ninéfescha et de la qualité des soins, qu'ils ne trouvent sans doute pas sur place à l'hôpital de Kedougou. Un système de navette s'est mis en place avec des transports collectifs amenant les citadins le matin et les reconduisant dans la journée ou le soir.

\section{Discussion et conclusion}

Nous avons étudié l'influence de la construction d'un hôpital moderne au cœur d'une région rurale du Sénégal mal équipée jusqu'alors, la zone de Bandafassi. Ce nouvel équipement avait pour premier objectif de faire reculer la mortalité maternelle, très élevée dans la région, en offrant des soins de haut niveau notamment en obstétrique. Les données de surveillance démographique montrent que la mortalité maternelle n'a pas baissé de façon sensible après l'ouverture de l'hôpital, du moins dans les quelques années qui ont suivi. Nous avons mené plusieurs enquêtes sur les comportements de recours aux soins et l'utilisation que les habitants faisaient de cet équipement, notamment en cas de grossesse et d'accouchement, pour mieux comprendre les raisons de cet échec et, de manière plus générale, les facteurs en cause dans la sous-utilisation des services de santé dans certaines régions d'Afrique subsaharienne.

Notre analyse montre que les villageois ont, dans l'ensemble, peu recours à l'hôpital quatre ans après son ouverture. La plupart des femmes ne s'y rendent pas en visite prénatale et ne vont pas y accoucher, ceci malgré des campagnes d'information incitatives. Les responsables de l'hôpital attribuent cet échec aux villageois, et notamment à leurs traditions. Les entretiens que nous avons menés montrent que le problème vient plutôt d'une inadéquation entre les conditions et l'environnement de l'offre de soins de l'hôpital de Ninéfescha (lieux d'accueil, mise en confiance, pratiques thérapeutiques) et les besoins. Un manque de coordination avant la mise en place de programmes ou d'infrastructures sanitaires entre la volonté politique, les responsables de santé et les populations concernées, mettent souvent en péril ces initiatives dues en grande partie à la non-prise en considération des facteurs socioéconomiques, culturels et géographiques (Jaffré et Olivier de Sardan, 2003 ; Kanté et al., 2008). Notre analyse détaillée des comportements en matière de recours aux soins pendant la grossesse et lors de l'accouchement dans la région de Bandafassi 
a permis de préciser le rôle de différents facteurs et d'examiner lesquels favorisent l'utilisation par les femmes enceintes de l'offre de soins médicale.

Le niveau d'instruction des femmes joue tant sur la probabilité qu'elles aillent en consultation prénatale que sur celle qu'elles accouchent en maternité, sachant que le niveau moyen est particulièrement faible dans la région, puisque $75 \%$ des femmes de l'échantillon (qui ont accouché dans les 12 derniers mois) n'ont jamais été à l'école. On retrouve ici l'influence déterminante de l'éducation des femmes dans l'usage qu'elles peuvent faire des services sanitaires (Caldwell, 1979 ; Hobcraft, 1993 ; Govindasamy et Ramesh, 1997). Ne disposant pas de données concernant le niveau d'instruction de tous les individus de cette population - ce type d'information n'est pas recueillie « en routine » par l'observatoire de population -, il ne nous est donc pas possible d'étudier les différences de mortalité maternelle selon le niveau d'instruction des femmes, de leur conjoint ou du chef de famille.

Pour ce qui est de l'âge de la femme et du rang de naissance à Bandafassi, les jeunes femmes et les primipares vont davantage en visite prénatale et accouchent plus fréquemment en maternité que les autres. L'accouchement d'une primipare présente plus fréquemment des difficultés que celui d'une autre femme, notamment quand elle est très jeune. Les femmes avaient en moyenne 18,5 ans à leur premier accouchement au début des années 2000 (tableau annexe 1 ; Guyavarch, 2003). Laccouchement d'une primipare donnerait donc lieu plus fréquemment à des complications amenant à un transfert de l'accouchée en maternité. Mais par ailleurs, anticipant d'éventuelles difficultés du fait justement de leur jeune âge et de leur manque d'expérience, les familles pourraient conduire plus souvent les primipares en maternité pour qu'elles accouchent en présence d'une aide qualifiée. Les comportements sanitaires durant la grossesse et lors de l'accouchement changent probablement plus vite chez les jeunes femmes, souvent plus instruites que leurs aînées. Il serait intéressant de continuer à suivre cette population afin d'observer la vitesse de ces changements ${ }^{(16)}$.

Les probabilités d'aller en visite prénatale et d'accoucher en maternité varient également selon le village de résidence et son éloignement par rapport aux postes de santé. Les femmes qui habitent à proximité du dispensaire de Bandafassi recourent davantage aux soins médicaux pendant la grossesse et lors de l'accouchement. En 2006-2007, toutes les femmes enceintes résidant à moins de $5 \mathrm{~km}$ du dispensaire de Bandafassi ont effectué au moins une visite prénatale et $80 \%$ d'entre elles ont accouché en maternité. En revanche, la plus ou moins grande proximité de l'hôpital de Ninéfescha ne joue pas. Seules 70 \% des femmes vivant à moins de $5 \mathrm{~km}$ de l'hôpital de Ninéfescha ont effectué une consultation prénatale, et moins de $10 \%$ ont accouché en maternité (tableau

(16) Il est probable que le nouvel hôpital finisse par avoir un effet à moyen terme, dans les dix à vingt années. Mais son ambition était de réduire rapidement la mortalité maternelle, ce que l'on est effectivement en droit d'attendre d'investissements de ce type. 
annexe 4). Si le recours aux soins est fonction en général de l'accessibilité géographique (Kroeger, 1983 ; Vigneron, 2001) ou de la proximité et la disponibilité d'un service (Stock, 1983 ; Fosu, 1989 ; Ronsmans et al., 2003 ; Souares et al., 2005), ces facteurs ne semblent pas jouer dans le cas de l'hôpital de Ninéfescha. La sous-utilisation de l'hôpital de Ninéfescha n'est pas causée en premier lieu par la distance. Les croyances populaires sur l'implantation d'une nouvelle infrastructure de soins ou certaines pratiques thérapeutiques dans les établissements de santé peuvent jouer un rôle et conduire à leur sous-fréquentation ou leur désaffection par les patients (Jaffré et Olivier de Sardan, 2003 ; Vidal et al., 2005). Une analyse plus détaillée des pratiques thérapeutiques à l'hôpital de Ninéfescha pourrait donc fournir des éléments d'explication complémentaires.

La distance euclidienne ne reflète qu'imparfaitement la plus ou moins grande proximité entre deux lieux, et il aurait été utile de disposer ici de mesures de temps de déplacement. En leur absence, comme le relief est la principale difficulté dans la zone d'étude, nous avons pris en compte la localisation des villages, en plaine ou en altitude. Cette localisation joue un rôle, car les femmes résidant dans les villages de plaine se rendent davantage à la consultation prénatale et accouchent plus souvent en maternité que les autres.

L'appartenance ethnique a également une influence - les femmes bedik consultent moins souvent que les femmes peul et malinké pendant la grossesse. Ceci est en partie lié à la localisation de leurs villages, plus souvent en altitude et difficiles d'accès, et de ce fait, moins souvent visités par l'infirmier, sachant qu'une partie importante des visites prénatales ont lieu au moment de ces visites. Le facteur déterminant n'est donc pas l'ethnie en elle-même, mais la localisation du village.

La part des femmes bénéficiant de consultations prénatales a augmenté fortement à Bandafassi au cours des dernières années, sans que ceci soit accompagné d'une diminution sensible de la mortalité maternelle. Ce résultat confirme les observations faites ailleurs en Afrique de l'Ouest (de Bernis et al., 2000 ; Saizonou et al., 2006), et conforte l'hypothèse selon laquelle le risque de mort maternelle est plus lié aux conditions de l'accouchement qu'au fait d'avoir bénéficié ou non d'une ou plusieurs consultations prénatales. Ce constat est d'ailleurs en partie à l'origine de la construction de l'hôpital de Ninéfescha dans la zone de Bandafassi, son objectif étant d'offrir à proximité immédiate des habitants de la région un service d'urgence obstétricale de grande qualité permettant notamment d'effectuer des césariennes. Le constat de notre étude est que la disponibilité de ce nouveau service n'a pas suffi à changer fondamentalement les conditions d'accouchement, la population n'y ayant que faiblement recours et continuant à accoucher à domicile dans la plupart des cas, ou lorsqu'elle se décide à accoucher en maternité, à aller encore au dispensaire local ou à l'hôpital de Kedougou, tout aussi éloignés sinon plus que l'hôpital de Ninéfescha, et moins bien équipés pour faire face à d'éventuelles urgences. 
Les instigateurs du nouvel hôpital croyaient s'être rapprochés des habitants en l'installant à proximité de leurs villages. Mais la population est restée à distance pour l'instant sans beaucoup changer ses habitudes. L'hôpital est peu attractif, on retrouve ici dans un contexte rural le constat établi en ville, pointant les difficiles relations entre soignés et soignants dans les hôpitaux des grandes capitales d'Afrique de l'Ouest (Jaffré et Olivier de Sardan, 2003). Lhôpital de Ninéfescha attire en revanche une clientèle de citadins plus fortunés que les villageois, qui n'hésite pas à faire l'aller-retour dans la journée depuis la ville voisine située à $35 \mathrm{~km}$ où il y a pourtant déjà un hôpital. Il aurait sans doute été plus pratique pour eux que le nouvel hôpital soit construit en ville, et les villageois n'y auraient sans doute pas beaucoup perdu car ils ne font qu'un usage restreint de l'hôpital de Ninéfescha. Le nouvel équipement illustre de façon exemplaire la nécessité de tenir compte des infrastructures existantes lorsqu'on en construit de nouvelles, de façon à ce qu'elles en soient complémentaires, et de prendre au préalable la mesure des attentes et des comportements en matière de recours aux soins, de façon à ce que l'offre soit adaptée. Si le cas de Bandafassi et de l'hôpital de Ninéfescha semble exemplaire, la question de la sous-utilisation se pose de façon générale pour beaucoup de nouvelles infrastructures sanitaires. 


\section{ANNEXES}

\section{Annexe 1. Évolution des quelques indicateurs de santé à Bandafassi, 1981-2006}

\begin{tabular}{|l|c|c|c|}
\hline \multicolumn{1}{|c|}{ Indicateur démographique } & \multicolumn{3}{c|}{ Période } \\
\cline { 2 - 4 } & $1981-1990$ & $1991-2000$ & $2000-2006$ \\
\hline Espérance de vie à la naissance (ans) (sexes confondus) & 39,2 & 46,1 & 52,8 \\
Risque pour un nouveau-né de mourir avant 5 ans & 361 & 278 & 189 \\
(5q0, \%) (sexes confondus) & 6,4 & 6,7 & 7,0 \\
Fécondité (nombre moyen d'enfants par femme) & 18,8 & 18,7 & 18,5 \\
Âge moyen à la première maternité (ans) & & \\
\hline Source : Base de données du site de suivi de Bandafassi (Pison et al., 2002 ; Kanté, 2009). \\
\hline
\end{tabular}

\section{Annexe 2. Caractéristiques des femmes ayant accouché à Bandafassi entre mars 2006 et mars-avril $2007(\mathrm{~N}=239)$}

\begin{tabular}{|c|c|c|c|c|c|}
\hline Variable & Modalité & $\%$ & Variable & Modalité & $\%$ \\
\hline \multirow[t]{2}{*}{$\begin{array}{l}\text { Appartenance } \\
\text { ethnique }\end{array}$} & $\begin{array}{l}\text { Peul } \\
\text { Bedik } \\
\text { Malinké }\end{array}$ & $\begin{array}{l}58,2 \\
23,4 \\
17,1\end{array}$ & $\begin{array}{l}\text { Fréquentation des } \\
\text { marchés } \\
\text { hebdomadaires }\end{array}$ & $\begin{array}{l}1 \text { fois par semaine } \\
1 \text { fois par mois } \\
\text { Jamais }\end{array}$ & $\begin{array}{l}10,9 \\
45,6 \\
43,5\end{array}$ \\
\hline & Autre & 1,3 & \multirow[b]{2}{*}{$\begin{array}{l}\text { Utilisation du } \\
\text { téléphone }\end{array}$} & 1 fois par semaine & 5,0 \\
\hline Religion & $\begin{array}{l}\text { Musulmane } \\
\text { Catholique }\end{array}$ & $\begin{array}{l}75,3 \\
24,7\end{array}$ & & $\begin{array}{l}1 \text { fois par mois } \\
\text { Jamais }\end{array}$ & $\begin{array}{l}41,4 \\
53,6\end{array}$ \\
\hline \multirow[t]{2}{*}{$\begin{array}{l}\text { Statut } \\
\text { matrimonial }\end{array}$} & $\begin{array}{l}\text { Monogame } \\
\text { Polygame }\end{array}$ & $\begin{array}{l}52,7 \\
43,5\end{array}$ & $\begin{array}{l}\text { Itinéraire } \\
\text { migratoire } \\
\text { (1 mois en ville) }\end{array}$ & $\begin{array}{l}\text { Oui } \\
\text { Non }\end{array}$ & $\begin{array}{l}54,8 \\
45,2 \\
\end{array}$ \\
\hline & Pas en union & 3,8 & \multirow[b]{2}{*}{ Chef de carré } & Père / Beau-père & 51,1 \\
\hline \multirow[t]{2}{*}{ Groupe d'âges } & $\begin{array}{l}\text { Moins de } 24 \text { ans } \\
\text { Entre } 24 \text { et } 32 \text { ans }\end{array}$ & $\begin{array}{l}34,7 \\
33,9\end{array}$ & & $\begin{array}{l}\text { Mari } \\
\text { Autre }\end{array}$ & $\begin{array}{l}29,7 \\
19,2\end{array}$ \\
\hline & 33 ans et plus & 31,4 & \multirow[b]{2}{*}{$\begin{array}{l}\text { Équipement du } \\
\text { ménage }\end{array}$} & Plus équipé & 8,4 \\
\hline \multirow[t]{2}{*}{$\begin{array}{l}\text { Pratique de la } \\
\text { lecture }\end{array}$} & $\begin{array}{l}\text { Très bien } \\
\text { Un peu }\end{array}$ & $\begin{array}{r}5,5 \\
34,7\end{array}$ & & $\begin{array}{l}\text { Moins équipé } \\
\text { Peu équipé }\end{array}$ & $\begin{array}{l}57,3 \\
34,3\end{array}$ \\
\hline & Pas du tout & 59,8 & \multirow{2}{*}{$\begin{array}{l}\text { Agent de santé } \\
\text { dans le village }\end{array}$} & Oui & 55,6 \\
\hline \multirow{2}{*}{$\begin{array}{l}\text { Fréquentation de } \\
\text { I'école publique }\end{array}$} & Oui & 24,3 & & Non & 44,4 \\
\hline & Non & 75,7 & \multirow[b]{2}{*}{$\begin{array}{l}\text { Système de santé } \\
\text { du village et } \\
\text { proximité du } \\
\text { dispensaire public } \\
\text { de Bandafassi }\end{array}$} & Privé & 28,0 \\
\hline $\begin{array}{l}\text { Fréquentation des } \\
\text { cours } \\
\text { d'alphabétisation }\end{array}$ & $\begin{array}{l}\text { Oui } \\
\text { Non }\end{array}$ & $\begin{array}{l}32,2 \\
67,8\end{array}$ & & $\begin{array}{l}\text { Public à moins de } \\
15 \mathrm{~km} \\
\text { Public à plus de } \\
15 \mathrm{~km} \text { ou en altitude }\end{array}$ & $\begin{array}{l}33,9 \\
38,1\end{array}$ \\
\hline Écoute de la radio & $\begin{array}{l}1 \text { fois par jour } \\
1 \text { fois par semaine } \\
\text { Jamais }\end{array}$ & $\begin{array}{l}65,1 \\
19,3 \\
15,6\end{array}$ & $\begin{array}{l}\text { Position du village } \\
\text { par rapport à } \\
\text { l'hôpital de } \\
\text { Ninéfescha }\end{array}$ & $\begin{array}{l}\text { Moins de } 15 \mathrm{~km} \\
\text { Entre } 15 \text { et } 30 \mathrm{~km} \\
\text { Altitude ou } \\
\text { plus } 30 \mathrm{~km}\end{array}$ & $\begin{array}{l}21,7 \\
31,0 \\
47,3\end{array}$ \\
\hline
\end{tabular}




\section{Annexe 3. Soins pendant la grossesse uniquement chez les femmes ayant effectué une visite prénatale entre mars 2006 et mars-avril $2007(\mathrm{~N}=183)$}

\begin{tabular}{|c|c|c|c|c|c|}
\hline Variable & Modalité & $\%$ & Variable & Modalité & $\%$ \\
\hline \multirow[t]{2}{*}{$\begin{array}{l}\text { Personnel } \\
\text { de santé } \\
\text { consulté }\end{array}$} & $\begin{array}{l}\text { Infirmier de Bandafassi }{ }^{(a)} \\
\text { Infirmier de la mission } \\
\text { catholique }^{(b)}\end{array}$ & $\begin{array}{l}63,4 \\
17,5\end{array}$ & A reçu une injection & $\begin{array}{l}\text { Oui } \\
\text { Non }\end{array}$ & $\begin{array}{r}94,0 \\
6,0\end{array}$ \\
\hline & $\begin{array}{l}\text { Ninéfescha }^{(c)} \\
\text { Autre }^{(d)}\end{array}$ & $\begin{array}{r}10,4 \\
8,7\end{array}$ & $\begin{array}{l}\text { Injection lors de la } \\
\text { grossesse précédente } \\
(\mathrm{N}=159)\end{array}$ & $\begin{array}{l}\text { Oui } \\
\text { Non }\end{array}$ & $\begin{array}{l}88,0 \\
12,0\end{array}$ \\
\hline \multirow[t]{2}{*}{$\begin{array}{l}\text { Lieu de la } \\
\text { consultation } \\
\text { prénatale }\end{array}$} & $\begin{array}{l}\text { Village } \\
\text { Bandafassi }^{(a)} \\
\text { Ninéfescha }^{(c)}\end{array}$ & $\begin{array}{r}43,7 \\
37,7 \\
9,4\end{array}$ & $\begin{array}{l}\text { Raison de la } \\
\text { non-consultation } \\
(\mathrm{N}=38)\end{array}$ & $\begin{array}{l}\text { Manque d'argent } \\
\text { Manque de temps } \\
\text { Autre }\end{array}$ & $\begin{array}{l}71,1 \\
13,2 \\
15,7\end{array}$ \\
\hline & Autre $\mathrm{b}^{(\mathrm{b})(\mathrm{d})}$ & 9,2 & \multirow{3}{*}{\multicolumn{3}{|c|}{$\begin{array}{l}\text { (a) Dispensaire public de Bandafassi. } \\
\text { (b) Dispensaire privé de la mission catholique Kedougou. } \\
\text { (c) Hôpital privé de Ninéfescha. } \\
\text { (d) Hôpital public de Kedougou ou dispensaire public } \\
\text { de Tomborokoto. }\end{array}$}} \\
\hline $\begin{array}{l}\text { Nombre } \\
\text { de visites } \\
\text { prénatales } \\
\text { effectuées }\end{array}$ & $\begin{array}{l}1 \\
2 \text { et } 3 \\
4 \text { et plus } \\
\text { Ne sait pas }\end{array}$ & $\begin{array}{r}13,1 \\
51,9 \\
33,3 \\
1,7\end{array}$ & & & \\
\hline $\begin{array}{l}\text { Connaît le rôle } \\
\text { des injections }\end{array}$ & $\begin{array}{l}\text { Oui } \\
\text { Non }\end{array}$ & $\begin{array}{l}45,4 \\
54,6\end{array}$ & & & \\
\hline
\end{tabular}




\section{Annexe 4. Facteurs influençant la consultation prénatale ou l'accouchement en maternité à Bandafassi, analyse univariée}

\begin{tabular}{|c|c|c|c|c|}
\hline Variable & Modalité & Effectif & $\begin{array}{l}\text { Consultation } \\
\text { prénatale }(\%)\end{array}$ & $\begin{array}{c}\text { Accouchement } \\
\text { en maternité } \\
(\%)\end{array}$ \\
\hline \multicolumn{5}{|c|}{ Caractéristiques de la femme } \\
\hline \multirow[t]{4}{*}{ Ethnie } & Bedik & 56 & $58,9 * * *$ & 12,5 \\
\hline & Malinké & 41 & $87,8 * * *$ & 12,2 \\
\hline & Peul & 139 & $90,6 * * *$ & 13,7 \\
\hline & Autre & 3 & 100,0 & 67,0 \\
\hline \multirow[t]{2}{*}{ Religion } & Musulmane & 180 & $89,9 * * *$ & 12,8 \\
\hline & Catholique & 59 & $61,7 * \star *$ & 16,7 \\
\hline \multirow[t]{2}{*}{ Groupe d'âges } & Moins de 24 ans & 83 & $90,4 * *$ & $19,3^{*}$ \\
\hline & 24 ans et plus & 156 & $78,9 * *$ & $10,9 *$ \\
\hline \multirow[t]{2}{*}{ Rang de la grossesse } & $1^{\text {ère }}$ grossesse & 24 & 87,5 & $37,5^{* * *}$ \\
\hline & $2^{\mathrm{e}}$ et plus & 215 & 82,3 & $11,2 * * *$ \\
\hline \multirow[t]{3}{*}{ Statut matrimonial } & Monogame & 126 & 81,7 & 16,7 \\
\hline & Polygame & 104 & 85,6 & 10,6 \\
\hline & Seule & 9 & 66,7 & $11,1^{(\mathrm{a})}$ \\
\hline \multirow{2}{*}{$\begin{array}{l}\text { Fréquentation } \\
\text { de l'école coranique }\end{array}$} & Oui & 132 & $91,7 * * *$ & 15,1 \\
\hline & Non & 107 & $72,0 * * *$ & 12,1 \\
\hline \multirow{2}{*}{$\begin{array}{l}\text { Fréquentation } \\
\text { de l'école publique }\end{array}$} & Oui & 58 & 89,7 & $25,9 * * *$ \\
\hline & Non & 181 & 80,7 & $9,9 * * *$ \\
\hline \multirow{3}{*}{$\begin{array}{l}\text { Dernière classe } \\
\text { fréquentée }\end{array}$} & Aucune & 181 & $80,7 * *$ & $9,9 * * *$ \\
\hline & Classe d'initiation $(\mathrm{Cl})$ et $\mathrm{CP}$ & 26 & $76,9 * *$ & $11,5 * * *$ \\
\hline & Supérieure au cours préparatoire (CP) & 32 & $100,0 * *$ & $37,5 * * *$ \\
\hline \multirow{2}{*}{$\begin{array}{l}\text { Fréquentation des cours } \\
\text { d'alphabétisation }\end{array}$} & Oui & 77 & $90,9 * *$ & 14,3 \\
\hline & Non & 162 & 79,0 ** & 13,6 \\
\hline \multirow[t]{2}{*}{ Pratique de la lecture } & Sait lire & 96 & $96,9 * * *$ & 17,7 \\
\hline & Ne sait pas lire & 143 & $73,4^{* * *}$ & 11,2 \\
\hline \multirow{2}{*}{$\begin{array}{l}\text { Fréquentation des } \\
\text { marchés hebdomadaires }\end{array}$} & Oui & 135 & $78,5 * *$ & $8,1 * *$ \\
\hline & Non & 104 & $88,5^{* *}$ & $21,1 * *$ \\
\hline \multirow[t]{2}{*}{ Utilisation du téléphone } & Oui & 111 & $88,3 * *$ & $23,4 * * *$ \\
\hline & Non & 128 & 78,1 ** & $5,5 * * *$ \\
\hline \multirow[t]{2}{*}{ Écoute de la radio } & Oui & 158 & $88,6 * * *$ & $16,5^{*}$ \\
\hline & Non & 81 & $71,6 * * *$ & $8,6^{*}$ \\
\hline \multirow{2}{*}{$\begin{array}{l}\text { Séjour en ville d'au } \\
\text { moins un mois }\end{array}$} & Oui & 131 & 86,3 & 16,1 \\
\hline & Non & 81 & 78,7 & 11,1 \\
\hline \multirow[t]{2}{*}{ Consultation prénatale } & Oui & 198 & - & 15,2 \\
\hline & Non & 41 & - & $7,3^{(a)}$ \\
\hline \multirow{2}{*}{$\begin{array}{l}\text { Lieu de consultation } \\
\text { prénatale }\end{array}$} & Oui & 189 & - & 15,4 \\
\hline & Non & 50 & - & 8,0 \\
\hline \multirow{2}{*}{$\begin{array}{l}\text { Complication à } \\
\text { I'accouchement }\end{array}$} & Oui & 43 & - & $30,2 * * *$ \\
\hline & Non & 196 & - & $10,2 * * *$ \\
\hline \multicolumn{5}{|c|}{ Caractéristiques du ménage } \\
\hline \multirow[t]{2}{*}{ Type de ménage } & Équipé & 157 & $87,9 * * *$ & 14,0 \\
\hline & Peu équipé & 82 & $73,2 * * *$ & 13,4 \\
\hline \multirow[t]{2}{*}{ Chef de carré } & Mari ou conjoint & 71 & 87,3 & $21,1 * *$ \\
\hline & Autre & 168 & 81,0 & $10,7 * *$ \\
\hline \multirow{2}{*}{$\begin{array}{l}\text { Nombre d'enfants } \\
\text { de moins de } 10 \text { ans }\end{array}$} & Moins de 3 & 158 & 83,5 & $18,4 * * *$ \\
\hline & Plus de 3 & 81 & 81,5 & $4,9^{(a) * * *}$ \\
\hline
\end{tabular}




\section{Annexe 5. Relations entre les différentes variables étudiées}

\section{Relation entre ethnie et religion (test de Pearson)}

\begin{tabular}{|l|c|}
\hline \multicolumn{1}{|c|}{ Variable } & \multicolumn{1}{c|}{ Ethnie } \\
\hline Religion & $\mathrm{R}=-0,84^{* * *}$ \\
\hline $\begin{array}{l}\text { Significativité statistique : }{ }^{* * *} \mathrm{p}<0,0001 . \\
\text { Source : Enquête sur le recours aux soins à Bandafassi, } 2007 .\end{array}$ \\
\hline
\end{tabular}

Relation entre les différentes caractéristiques sociodémographiques des femmes (test de $\chi^{2}$ à 4 cases)

\begin{tabular}{|l|l|c|c|}
\hline \multicolumn{2}{|c|}{ Variable } & Valeur du $\chi^{2}$ & $p$-value \\
\hline Pratique de la lecture & École publique & 17,8 & $* * *$ \\
& École coranique & 55,1 & $* * *$ \\
& Alphabétisation & 4,0 & $*$ \\
\hline Rang de naissance & Groupe d'âges & 50,1 & $* * *$ \\
\hline Écoute de la radio & Utilisation du téléphone & 18,3 & $* * *$ \\
& Séjour hors de la région supérieur à 1 mois & 11,8 & $* * *$ \\
& Fréquentation du marché hebdomadaire & 0,8 & $0,37^{\text {n.s. }}$ \\
\hline \multicolumn{2}{|l}{ Significativité statistique : ${ }^{* * *} p<0,0001 ; * * p<0,001 ; * p<0,01 ;$ n.s. non significatif. } \\
\multicolumn{2}{|l}{ Source : Enquête sur le recours aux soins à Bandafassi, 2007.} & \\
\hline
\end{tabular}

\section{Relation entre les caractéristiques du ménage (test de $\chi^{2}$ à 4 cases)}

\begin{tabular}{|l|l|c|c|}
\hline \multicolumn{2}{|c|}{ Variable } & Valeur du $\chi^{2}$ & p-value \\
\hline Équipement & Taille du ménage & 0,26 & $0,61^{\text {n.s. }}$ \\
du ménage & Lien avec le chef de carré (conjoint ou autre) & 1,17 & $0,27^{\text {n.s. }}$ \\
\hline
\end{tabular}

Significativité statistique : ${ }^{\text {n.s. }}$ non significatif.

Source : Enquête sur le recours aux soins à Bandafassi, 2007.

\section{Relation entre les différentes caractéristiques sanitaires du village} (test de Pearson)

\begin{tabular}{|l|c|}
\hline \multicolumn{1}{|c|}{ Variable } & $\begin{array}{c}\text { Situation du village par rapport } \\
\text { au dispensaire de Bandafassi }\end{array}$ \\
\hline $\begin{array}{l}\text { Situation du village par rapport à l'hôpital de Ninéfescha } \\
\text { Présence d'un agent de santé dans le village }\end{array}$ & \multicolumn{1}{|c|}{$\mathrm{R}=0,20 * *$} \\
\hline $\begin{array}{l}\text { Significativité statistique : *** } p<0,0001 ; * * \\
\text { Source : Enquête sur le recours aux soins à Bandafassi, } 2007 .\end{array}$ \\
\hline
\end{tabular}




\section{Annexe 6. Facteurs influençant la consultation prénatale}

\section{Analyse par régression multivariée}

\begin{tabular}{|c|c|c|c|}
\hline Variable & Modalité & OR [IC à 95\%] & $p$ \\
\hline Lecture & Sait lire / Ne sait pas lire & $5,2[1,5-18,6]$ & ** \\
\hline \multirow{5}{*}{$\begin{array}{l}\text { Appartenance ethnique } \times \\
\text { Système de santé du village et } \\
\text { situation par rapport au dispensaire } \\
\text { de Bandafassi }\end{array}$} & $\begin{array}{l}\text { Bedik du secteur privé / Bedik du } \\
\text { secteur public en altitude }\end{array}$ & $2,7[0,8-9,9]$ & n.s. \\
\hline & $\begin{array}{l}\text { Bedik du secteur public en } \\
\text { plaine }{ }^{(b)} / \text { Bedik du secteur public } \\
\text { en altitude }\end{array}$ & $9,0[1,0-83,9]$ & * \\
\hline & $\begin{array}{l}\text { Malinke du secteur privé / Bedik } \\
\text { du secteur public en altitude }\end{array}$ & $8,5[2,6-28,1]$ & $* * *$ \\
\hline & $\begin{array}{l}\text { Peul du secteur public en } \\
\text { altitude / Bedik du secteur public } \\
\text { en altitude }\end{array}$ & $5,1[1,9-13,3]$ & *** \\
\hline & $\begin{array}{l}\text { Peul du secteur public en plaine / } \\
\text { Bedik du secteur public en } \\
\text { altitude }\end{array}$ & $14,6[2,4-113,9]$ & *** \\
\hline \multicolumn{4}{|c|}{$\begin{array}{l}\text { Nombre d'observations }=239 \text {. } \\
\text { Significativité statistique: }{ }^{* *} p<0,01 ; * * p<0,05 ; * p<0,10 ; n s=\text { non significatif. } \\
\text { (a) Public en altitude ou à plus de } 15 \mathrm{~km} \text { du dispensaire de Bandafassi } \\
\text { (b) Public en plaine et à moins de } 15 \mathrm{~km} \text { du dispensaire de Bandafassi }\end{array}$} \\
\hline \multicolumn{4}{|c|}{$\begin{array}{l}\text { Note : Ce tableau ne présente que les variables qui restent significatives dans le modèle après } \\
\text { ajustement (option descendante) sur les autres variables (groupe d'âges, écoute de la radio, type de } \\
\text { ménage, situation du village par rapport a l'hôpital de Ninéfescha). }\end{array}$} \\
\hline \multicolumn{4}{|c|}{ Source : Enquête sur le recours aux soins à Bandafassi, 2007.} \\
\hline
\end{tabular}




\section{RÉFÉRENCES}

BARBIERI Magali, VAlLin Jacques, 1996, « Les conséquences de la crise économique africaine sur l'évolution de la mortalité », in Coussy Jean, Vallin Jacques (dir.), Crise et population en Afrique. Crises économiques, politiques d'ajustement et dynamiques démographiques, Paris, Ceped, Les études du Ceped, nº 13, p. 319-343.

DE Bernis Luc, Dumont Alexandre, Bouillin Dominique, Gueye Abdoulaye, DOMPNIER Jean-Pierre, BOUVIER-COLLE Marie-Hélène, 2000, « Maternal morbidity and mortality in two different populations of Senegal: A prospective study (MOMA survey) », British Journal of Obstetrics and Gynaecology, 107(1), p. 68-74.

CALDWELL John, 1979, «Education as a factor in mortality decline: An examination of Nigerian data », Population studies, 33(3), p. 395-413.

CANTRELLE Pierre, LOCOH Thérèse, 1990, Facteurs culturels et sociaux de la santé en Afrique de l'Ouest, Paris, Ceped, Les dossiers du Ceped, n 10, 36 p.

CANTRElle Pierre, THONNEAU Patrick, TOURÉ Boubacar, 1992, Mortalité maternelle : deux études communautaires en Guinée, Paris, Ceped, Les dossiers du Ceped, n²0, 43 p.

COMMEYRAs Christophe, NDO Jean-Rolin, 2003, « Études de l'accessibilité et des déterminants de recours aux soins et aux médicaments pour les populations du Cameroun ", communication présentée au Colloque Les journées des économistes français de la santé : Santé et développement, organisé par Cerdi, Clermont-Ferrand, France, $21 \mathrm{p}$.

DesgréEs du Loû Annabel, Pison Gilles, SAmb Badara, TrAPe Jean-François, 1996, « L'évolution des causes de décès d'enfants en Afrique : une étude de cas au Sénégal avec la méthode d'autopsie verbale », Population, 51(4-5), p. 845-882.

DiAllo Yveline, 2003, « Pauvreté et maladie », in Jaffré Yannick, Olivier de Sardan Jean-Pierre (dir.), Une médecine inhospitalière. Les difficiles relations entre soignants et soignés dans cinq capitales d'Afrique de l'Ouest, Paris, Karthala, p. 157-216.

Énel Catherine, PISON Gilles, LefEbvre Monique, 1993, « De l'accouchement traditionnel à l'accouchement moderne au Sénégal », Cahiers santé, 3, p. 441-446.

FOSU Gabriel, 1989, « Access to health care in urban areas of developing countries », Journal of Health and Social Behavior, 30, p. 398-411.

GARENNE Michel, FOnTAINE Olivier, 1988, « Enquête sur les causes probables de décès en milieu rural sénégalais », in Vallin Jacques, D’Souza Stan, Palloni Alberto (dir.), Mesure et analyse de la mortalité. Nouvelles approches, Paris, Ined/Puf, Cahier $\mathrm{n}^{\circ} 119$, p. 123-141.

GASTiNEAU Bénédicte, 2003, « Offre de soins, recours aux soins et santé des femmes en Tunisie », in Arber Sara, Khlat Myriam (eds.), Social and Economic Patterning of Health among Women [Les facteurs sociaux et économiques de la santé des femmes], Paris, Cicred, p. 407-424.

GOMila Jacques, Clarkson May, 1983, «Éléments pour une ethnographie du mariage et de la reproduction chez les Bedik (Sénégal oriental) », Anthropologie et sociétés, 7(2), p. 131-163.

GOVINDASAMY Pavalavalli, RAMESH M. B., 1997, Maternal Education and the Utilization of Maternal and Child Health Services in India, Mumbai, National Family Health Survey Subject Reports, $\mathrm{n}^{\circ}$ 5, International Institute for Population Sciences, $28 \mathrm{p}$. 
GUYAVARCH Emmanuelle, 2003, Démographie et santé de la reproduction en Afrique subsaharienne. Analyse des évolutions en cours. Une étude de cas : l'observatoire de population de Bandafassi (Sénégal), Paris, Muséum national d'histoire naturelle, Thèse de doctorat de démographie, 349 p. + annexes.

Hill Althea, 1989, «La mortalité des enfants : niveau actuel et évolution depuis 1945 », in Pison Gilles, Van De Walle Etienne, Sala-Diakanda Mpembele (dir.), Mortalité et société en Afrique au sud du Sahara, Paris, Ined/Puf, Cahier no 124, p. 13-34.

HOBCRAFT John, 1993, «Women's education, child welfare and child survival: A review of the evidence », Health Transition Review, 3(2), p. 159-176.

JAFFRÉ Yannick, OliVIER DE SARDAN Jean-Pierre (dir.), 2003, Une médecine inhospitalière. Les difficiles relations entre soignants et soignés dans cinq capitales d'Afrique de l'Ouest, Paris, Karthala, 449 p.

KANTÉ Almamy Malick, 2007, « Un hôpital, mais pas de malade ! Les contraintes de l'accès aux soins de santé en milieu rural sénégalais », in Actes du $1^{\text {er }}$ symposium international de Dakar, Stratégies de population et stratégies de développement : convergences ou divergences?, Dakar, Université de Dakar (Sénégal), p. 185-206.

KANTÉ Almamy Malick, 2009, Santé et mortalité des mères et des enfants à Bandafassi (Sénégal) : niveaux, tendances et influence du recours aux soins, Paris, Université Paris I - Panthéon-Sorbonne, Thèse de doctorat de démographie, 600 p.

KANTÉ Almamy Malick, GUYAVARCH Emmanuelle, PISON Gilles, 2008. « Pourquoi la mortalité maternelle ne diminue-t-elle pas plus vite en Afrique malgré l'amélioration de l'offre sanitaire? Choc des cultures ou mauvaise organisation sanitaire ? L'expérience de la population rurale de Bandafassi au Sénégal », in Actes du $X V^{\mathfrak{e}}$ colloque international de l'Association internationale des démographes de langue française (AIDELF), Démographie et cultures, Québec (Canada), p. 335-356.

KROEGER Axel, 1983, «Anthropological and socio-medical health care research in developing countries », Social Sciences and Medicine, 17(3), p. 147-161.

LEROY Odile et GARENNE Michel, 1989, « La mortalité par tétanos néonatal : la situation à Niakhar au Sénégal », in Pison Gilles, Van De Walle Etienne, Sala-Diakanda Mpembele (dir.), Mortalité et Société en Afrique au sud du Sahara, Paris, Ined/Puf, Cahier no 124, p. 153-167.

DE Lestrange Marie-Thérèse, PASSOT-GuÉVARA Béatrice, 1981, « De la grossesse au sevrage, chez les Bassari, les Peul et les Boin de Salémata (Sénégal oriental) », Sociétés contemporaines, 55, p. 21-42.

MOSLEY Henri, CHEN Lincoln, 1984, « An analytical framework for the study of child survival in developing countries », Population and Development Review, 10(sup.), p. 25-45.

NATIONS UNIES, 2009, World Population Prospects: The 2008 Revision, New York, Division de la Population, Département des affaires économiques et sociales des Nations unies.

NDIAYE Salif, AYAD Mohamed, 2006, Enquête démographique et de santé au Sénégal (EDS-IV) 2005, Dakar, Ministère de la santé et de la prévention médicale, Centre de recherche pour le développement humain, Calverton, ORC Macro, 487 p.

OMS, 1977, Classification internationale des maladies. Révision 1975, 1, Genève, Organisation mondiale de la santé.

OMS, 1993, Classification internationale des maladies et des problèmes de santé connexes. Dixième révision, 1, Genève, Organisation mondiale de la santé.

OMS, 2006, Rapport sur la santé dans le monde 2006. Travailler ensemble pour la santé, Genève, Organisation mondiale de la santé.

OMS, 2007, Maternal Mortality in 2005, Genève, WHO, UNICEF, UNFPA and The World Bank, 40 p. 
PALlONi Alberto, 1985, "Santé et lutte contre la mortalité en Amérique latine », in Vallin Jacques, Lopez Alan (dir.), La lutte contre la mort : influence des politiques sociales et des politiques de santé sur l'évolution de la mortalité, Paris, Ined/Puf, Cahier $\mathrm{n}^{\circ}$ 108, p. 447-475.

PISON Gilles, 1982, Dynamique d’une population traditionnelle : les Peul Bandé (Sénégal oriental), Paris, Ined/Puf, cahier n ${ }^{\circ}$ 99, 278 p.

PISON Gilles, 2006, « Les observatoires de population, outils pour étudier les changements démographiques et sanitaires dans les pays du Sud », in Caselli Graziella, Vallin Jacques, Wunch Guillaume (dir.), Démographie: analyse et synthèse, vol. 8, p. 125-150.

PISON Gilles, KODIO Belco, GUYAVARCH Emmanuelle, ETARD Jean-François, 2000, "La mortalité maternelle en milieu rural au Sénégal », Population, 55(6), p. 1003-1018.

PISON Gilles, GUYAVARCH Emmanuelle, SOKHNA Cheikh, 2002, « Bandafassi demographic surveillance system, Senegal », in INDEPTH network, Population and Health in Developing Countries, Ottawa, International Development Research Centre, p. 263-269.

Ronsmans Carine, Etard Jean Francois, Walraven Gijs, HoJ Lars, DumOnt Alexandre, DE BERNIS Luc, KODIO Belco, 2003. « Maternal mortality and access to obstetric services in West Africa », Tropical Medicine and International Health, 8(10), p. $940-948$.

SAizONOU Jacques, OUENDO Edgard-Marius, DujARdin Bruno, 2006, « Audit des décès maternels dans quatre maternités de référence du Bénin - qualité des soins d'urgence, causes et facteurs contributifs », African Journal of Reproductive Health, 10(3), p. 28-40.

Souares Aurélia, Chippaux Jean-Philippe, Le Hesran Jean-Yves, 2005, « Effets de l'amélioration de l'offre de soins sur l'activité d'un poste de santé en zone rurale au Sénégal », Revue deépidémiologie et de santé publique, 53(2), p. 143-151.

STOCK Robert, 1983, « Distance and the utilization of health facilities in rural Nigeria », Social Science and Medicine, 17(9), p. 563-570.

TAButin Dominique, SCHoumaker Bruno, 2004, « Démographie de l'Afrique au sud du Sahara des années 1950 aux années 2000 : synthèse des changements et bilan statistique », Population, 59(3-4), p. 521-622.

VIDAL Laurent, FALl Abdou Salam, GADOU Dakouri (dir.), 2005, Les professionnels de santé en Afrique de l'Ouest. Entre savoirs et pratiques, Paris, L'Harmattan, 328 p.

Vigneron Emmanuel, 2001, Distance et santé. La question de la proximité des soins, Paris, Puf, Médecine et société, Territoires et économie de la santé, 127 p.

Witter Sophie, Armar-Klemesu Margaret, Dieng Thierno, 2008, « National fee exemption schemes for deliveries: Comparing the recent experiences of Ghana and Senegal », in Richard Fabienne, Witter Sophie, De Brouwere Vincent, Reducing the Financial Barriers to Access to Obstetric Care, Studies in Health Services Organisation and Policy series, 24, Antwerp, ITG Press, p. 167-199. 


\section{Almamy Malick KANTÉ, Gilles PISON • LA MORTALITÉ MATERNELLE EN MILIEU RURAL} SÉNÉGALAIS. L'EXPÉRIENCE DU NOUVEL HÔPITAL DE NINÉFESCHA

En Afrique, plus qu'ailleurs, l'offre de soins reste insuffisante. La construction de nouvelles infrastructures sanitaires suffit-elle à faire progresser la santé de la population ? La question se pose lorsque de nouvelles installations sanitaires sont construites sans être suivies d'une amélioration rapide des indicateurs de santé. Comment alors expliquer la lenteur des changements ? Provient-elle de l'inadéquation entre l'offre et les besoins ? De " freins culturels » empêchant de nouveaux comportements de se diffuser ? Cet article examine les facteurs en cause dans le cas de l'implantation d'un hôpital moderne en 2003 au cœur d'une région rurale du Sénégal, jusqu'alors mal équipée. L'observation démographique suivie de la population pendant plusieurs décennies montre que la mortalité maternelle n'a pas baissé de façon sensible juste après l'ouverture de l'hôpital. Pour mieux comprendre les raisons de cet échec, plusieurs enquêtes ont été menées sur les comportements de recours aux soins et l'utilisation que les habitants faisaient de cet équipement, notamment en cas d'accouchement. Les villageois ont, dans l'ensemble, peu recours à l'hôpital quatre ans après son ouverture. La plupart des femmes ne s'y rendent pas en visite prénatale et ne vont pas y accoucher. Les responsables de l'hôpital attribuent cet échec aux villageois, et notamment à leurs traditions. Ces enquêtes montrent que le problème vient plutôt d'une inadéquation entre l'offre de soins de l'hôpital et les besoins.

\section{Almamy Malick Kanté, Gilles Pison • Maternal Mortality in Rural Senegal. The EXPERIENCE OF THE NEW NinÉfESCHA HOSPITAL}

Africa continues to suffer from an acute lack of healthcare provision. Is the construction of new healthcare facilities sufficient to improve the health of the population? This is certainly a question raised when the opening of a new hospital fails to bring about any rapid improvement in health indicators. It is difficult to account for the slowness of change here. Does it stem from a mismatch between supply and demand? Or from "cultural brakes" hindering the spread of modern ideas? This article investigates possible factors in the case of a modern hospital built in Bandafassi, a rural area of Senegal where facilities had previously been scarce. Data collected over several decades through demographic surveillance of the local population showed that there was no noticeable decline in maternal mortality immediately following the hospital's opening. To identify the reasons for this failure, we conducted several surveys of local people's health-seeking behaviour and the way they utilized this facility, notably for childbirth. Four years on, villagers were still making very little use of the hospital. Few women went there either for antenatal visits or to give birth. The hospital management ascribed this failure to the villagers themselves and, more particularly, to their traditions. These surveys, however, indicate that the heart of the problem lies in a mismatch between the services offered by the hospital and local people's actual needs.

\section{Almamy Malick Kanté, Gilles PISON • LA MORTALIDAD MATERNA EN EL MEDIO RURAL SENEGALÉS. LA EXPERIENCIA DEL NUEVO HOSPITAL DE NINEFESCHA}

En África, más que en otros países, la oferta sanitaria es todavía insuficiente ¿La construcción de nuevas estructuras sanitarias basta para mejorar la salud de la población? Esta pregunta se plantea cuando se observa que la construcción de nuevas instalaciones sanitarias no es seguida de una mejora rápida de los indicadores de salud ¿Cómo explicar en este caso la lentitud de los cambios? ¿Se trata de una inadecuación entre la oferta sanitaria y las necesidades de la población? ¿Hay « frenos culturales " que impiden la difusión de nuevos comportamientos? Este artículo examina los factores responsables en el caso de la implantación en 2003 de un hospital moderno en el centro mismo de una región rural de Senegal, hasta entonces mal equipada. La observación demográfica de la población durante varias décadas muestra que la mortalidad materna no ha disminuido de manera significativa inmediatamente después de la apertura del hospital. Para comprender mejor las razones de este fracaso, varias encuestas han sido realizadas sobre los comportamientos de recurso a la asistencia médica y la utilización que los habitantes hacían del hospital, particularmente en lo que concierne el parto. Cuatro años después de la apertura, los habitantes continúan haciendo poco uso del hospital. La mayor parte de las mujeres no acuden a él en visita prenatal ni van para dar a luz. Los responsables del hospital atribuyen este fracaso a los aldeanos y en particular a sus tradiciones. Sin embargo, las encuestas realizadas muestran que el problema proviene más bien de una inadecuación entre la oferta sanitaria y las necesidades de la población.

Mots-clés : Sénégal, mortalité maternelle, consultation prénatale, nouvelle offre sanitaire, observatoire de population.

Keywords: Senegal, maternal mortality, antenatal consultation, new healthcare provision, demographic surveillance. 